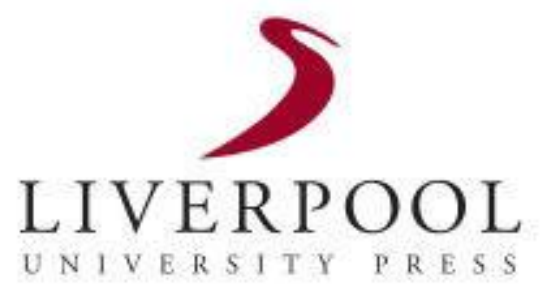

Fasciculus Morum: Foinse Chomónta do Phrós Cráifeach na Gaeilge sa 17ú hAois? Author(s): Tadhg Ó Dúshláine

Source: Studia Hibernica, No. 34 (2006-2007), pp. 111-122

Published by: Liverpool University Press

Stable URL: http://www.jstor.org/stable/40732134

Accessed: 27-06-2018 14:56 UTC

JSTOR is a not-for-profit service that helps scholars, researchers, and students discover, use, and build upon a wide range of content in a trusted digital archive. We use information technology and tools to increase productivity and facilitate new forms of scholarship. For more information about JSTOR, please contact support@jstor.org.

Your use of the JSTOR archive indicates your acceptance of the Terms \& Conditions of Use, available at http://about.jstor.org/terms 


\title{
Fasciculus Morum: Foinse Chomónta do Phrós Cráifeach na Gaeilge sa 17ú hAois? ${ }^{1}$
}

\author{
Tadhg Ó Dúshláine \\ (Ollscoil Náisiúnta na hÉireann, Má Nuad)
}

Is í an 17ú haois, aois mhór phrós na Gaeilge, prós reitriciúil, teagascach a bhfuil aidhmeanna cinnte caiticéise leis don chuid is mó, ach nach den gcaiticéis iarThriontach, faoi mar a leag Canisius amach í, ach mar fhorbairt agus mar dhaingniú ar an ábhar cráifeachta agus seanmóireachta a bhí fadbhunaithe ón mheánaois i leith. Bíodh a fhianaise sin ar thrí cinn de leabhair mhóra na tréimhse: SSA, TBGB agus PnamB. ${ }^{2}$ Seanmóirithe iomráiteacha a scríobh iad: Aodh Mac Aingil agus rá a bhéil fhéin againn mar fhianaise - 'ó nách léigthear dhúinn tré bhurba an pherseacuision forcheadal do dhénamh ó bheól' (94); b'fhéidir a áiteamh gur aon seanmóin mhór fhada amháin ar an mbás is ea TBGB, idir ábhar agus reitric; agus cnuasach óráidí ar na suáilcí agus na duáilcí is ea PnamB. Ní hamháin go bhfuil samplaí áirithe i gcuibhreann eatarthu ach tá scata den ábhar iontu le fáil sa Fasciculus Morum (FM anseo thíos), Leabhrán na mBéas, a tiomsaíodh le Proinsiasach Sasanach ag deireadh na 14ú haoise, mar áis seanmóireachta agus a bhí in úsáid choitianta ina dhiaidh sin, maraon leis an Liber Exemplorum, a chóirigh Proinsiasach Sasanach, lonnaithe in Éirinn, thart ar an mbliain 1275, an Speculum Laicorum, a chuir Proinsiasach Sasanach eile le chéile thart ar an dtréimhse chéanna, agus Les contes moralises de Nicole Bozon, frére mineur, a bhailigh Proinsiasach Sasanach eile fós go luath tar éis 1320 .

Is ar éigean má bhí an FM ina fhoinse dhíreach ag aon cheann de na bailiúcháin thuas, áfach, óir den bhreis agus dhá chéad exemplum ann, níl ach an t-aon cheann cinnte amháin - an ceann coitianta sin 'Breithiúnas Cambeses' - le fáil i gceann amháin de na cnuasaigh Ghaeilge. Ní hionann agus an bailiúchán cáiliúil eile sin na Fabulae agus Parabolae de chuid Odo de Cheritona (+1240), ón gcéad leath den dtrí haois agus a bhfuil cúig exemplum dá chuid le fáil i saothar an Chéitinnigh (dhá cheann in ESA, dhá cheann in TBGB agus ceann sa bhFF), ceann in SSA Mhic Aingil agus ceann i bPnamB. 'Sí aidhm an pháipéir seo, mar sin, ná a thaispeáint gur chuid d'oidhreacht choitianta na meánaoise cuid mhaith d'ábhar cráifeach (an chuid is fearr ó thaobh na litríochta, d'áiteoinn) an 17ú haois sa Ghaeilge agus nach aon fhás

\footnotetext{
'Leagan de chaint a tugadh ag an gComhdháil Cheilteach i mBonn, 2007.
}

${ }^{2}$ Féach deireadh na haiste seo do liosta na nod. 
thar oíche é ó Scoil na Nua-Ghaeilge sa Lováin, i mBordeaux ná áit ar bith eile a bhí ann. Is é sin le rá, go mba oidhre cuid mhaith den ábhar sin ar an 'failed renaissance' dá dtagraíonn Beryl Smalley ina sárleabhar English Friars and Antiquity in the Early XIV Century (London 1960).

\section{Scáthán Shacramuinte na hAithridhe}

Féachaimis ar dtús ar na samplaí san atá comónta don FM agus SSA:

1. An démond, cloch as cruaidhe do chlochuibh, maothuighthear í 7 do-níthear deaghobair uirre le fothrogadh fola do thabhairt dí. Croith, a Thiaghearna, braon dod dhiadhfhuil do dhoirtis san chronn, ar an gcroidhe cclochdha sa dá mhaothughadh dochum oibre na haithridhe. Do sgoltadh na cairrge a n-aimsir do pháisi ag caoineadh ciontadh gcomhuidheach; tabhair, a Thighearna, ar chruaidhcharnuig mo chroidhe-si sgoltadh tré dhoilghios 7 tré thuirsi fám peacadhuibh dísle féin. SSA 12

Et ideo talis bene comparatur adamanti qui secundum Ysidorum nulli cedit materie sicut ferro nec igne frangitur nec umquam incalescit, unde 'adamas' Grece, 'vis indomita' Latine dicitur; set dum sic invictus fuerit ferri ignisque contemptor, tamen sanguine yrcino maceratur. Revera sic verus paciens nulla vi ferri aut ignis iniuriarum frangitur aut dissolvitur, et tamen amore sanguinis Christi Iesu statim ad compassionem emollitur. FM 136

Tá sampla eile den tsamhail seo in FM agus é curtha síos d'Aguistín:

Et ponit [Abhaistín] exemplum: 'Adamas, inquit, inter mallium et ferrum non frangitur, et tamen hircino sanguine dissipatur. Sic (dicit ipse) vir aut mulier fortis, qui tribulacione non frangitur, in sanguine, hoc est in peccato Veneris, levius resolvitur et dissipatur.' FM 706

Tá curtha in iúl ag Aodh Mac Aingil i nóta bhun leathanaigh gur féidir an sampla seo a ríomh siar go Plinius agus Isadorus agus ní beag de theist ar bhríomhaireacht thraidisiún na cráifeachta dúchais go bhfaightear leagan de sa traidisiún béil:
A Íosa, a AonMic an Athar 's a Uain
'thug fíorfhuil do chroí istigh dár gceannach go crua, bí i m'dhídean, bí i m'choimhdeacht, bí i m'aice go buan, más luí dom, más suí, más seasamh, más suan.

Ísligh mo dhíoltas, m' fhearg is m' fhuath Is díbir na smaointe mallaithe uaim. Lig braon ó d'Naomh Spiorad beannaithe anuas A scaoilfidh an croí seo 'tá 'na charraig le cruas. ${ }^{3}$

${ }^{3}$ Diarmaid Ó Laoghaire, Ár bPaidreacha Dúchais (Baile Átha Cliath, 1975), uimh. 16,151 . 
Aithnítear sa dara sampla seo a leanas foinse na cráifeachta maoithní sin as ar eascair cleachtas na deabhóide don Chroí Ró-Naofa:

2. Adeir féin go bhfuil 'na sheasamh ag síorbhualadh dhoruis do chroidhe ag iarruidh osguilti. Apoc.3. SSA 16

Set modo ad ostium cordis nostri pulsat; Apocalipsis 3: "Ecce ego sto ad ostium et pulso..."FM 452

Ba mhór i gceist an téacs seo mar théama ag filí an Bhéarla sa 14ú haois agus tá sampla gleoite i gcló ag Carleton Brown in Religious Lyrics of the Fourteenth Century (Oxford, 1952), lch 86.

Scáthánaítear toscaí cíorthuathaileacha na linne i dtéama an bháis i litríocht Ghaeilge an 17ú haois agus tugann Mac Aingil roinn iomlán de SSA suas do scéalta uafásacha timpeall an téama chéanna:

3. Sompladha Diadha a n-Aghuidh na Muintire Chuireas an Aithrighe ar Cáirde, go Mórmhór go hAm an Bháis. C. 12

Scríobhuidh an t-athair beannuighthe Béda go raibhi duine uasal a Saxain fá mhúirnn mhóir ag an rígh Conredus tré a fheabhas do ghaisgiodhach ... [Li 5 Historiae Anglic., c. 14]

Admhaíonn Mac Aingil go gcuireann sé an scéal seo de chuid Bhéada síos d'aonghnó chun daoine a 'bhrostú' chun aithrí agus 'as é an t-ádhbhar cédna soin tug orumsa a chor a nGaoighilg chugad, a fhir éintíre' (1379-80). Ach cuireann sé faoi chló stíl na linne é faoi mar a dhein Thomas Stapleton ina aistriúchán sin ar shaothar Bhéada, The History of the Church of Englande (1565).

The familiar ease and picturesqueness of the prose ... the lyrical phrases ... introduced to highten the feeling ... the drawing of the character, which gives to the passage ... a concreteness and actuality which is none too common in historical writing. ${ }^{4}$

\section{Mar seo atá sa FM:}

Tercium malum quod evenit confiteri differentibus est adquisicio eterne pene. Unde narrat Beda De gestis Anglorum libro 5, capitulo 13, de quodam milite qui fuit cum Kynredo rege Merciorum, quem rex multum dilexit quia probus in armis extiterat valde... 488

\footnotetext{
${ }^{4}$ Féach A. C. Southern, Elizabethan Recusant Prose, 1559-1582 (London, 1950), 93-4.
} 
Cuid d'oidhreacht raibiléiseachas na meánaoise is ea an mugadh magadh reitriciúil a dheintí d'iarrachtaí mínithe agus leithscéil an phobail i gcúrsaí aithrí agus faoistine:

4. De quibus Bernardus: "Multis modis fiunt excusaciones in peccatis. Dicit enim aliquis se excusans, 'Non feci quid, aut saltem bene feci, aut si male feci non tamen multum male, aut si multum male non tamen mala intencione."'... FM 467

Set est advertendum, quod dolendum est, quod multi hiis diebus non sic faciunt, set fatue peccata sua excusant et in alios retorquent, et hoc multis modis: aliquando per consuetudinem, aliquando per societatem, aliquanodo Deo imputantes. Ibid. 474

\section{Mar seo atá ag Mac Aingil:}

Ní laghduigheann 7 ní mhéduigheann peacadh na meisgi a dhénamh san n-oidhche nó san ló, a dtigh Bhríain nó Mhurchaidh, gur bheanuis ré mnaoi Shaxanuidh nó Éirionnuidh. Ní laghduigheann 7 ní mhéduigheann peacadh na meisgi a dénamh san n-oidhche seach a dénamh san ló, nó a dénamh a dtigh Bhríain seach a dénamh a dtigh Mhurchaidh, 7 as ionann an peacadh luighi ré mnaoi nÉirionnuigh nó Shaxanaidh; ór ní holc bheith a dtigh Bhríain nó Mhurchaidh, ní hí holc oidhche ná lá, 7 , dá bhrígh sin, ní mhéduigh olc. An mheisgi amháin atá 'na peacadh, 7 an drúis, 7 as lór iad soin do rádh 7 gan teachd ar na haicídibh eili si ar énchor. SSA 87

\section{As traidisiún an vanitas vanitatum a fháisctear an sampla a leanas:}

5. Et mirum est quod homines tantum de amissione temporalium tristantur cum non sit nisi quedam umbra aut fumus, prout ait beatus Augustinus in quodam sermone dicens: " $O$, inquit, vita presens mundialis, que tantos decepisti et excecasti! Cum enim fugis, nichil es. Cum videris, umbra es. Cum exaltaris, fumus es. Stultis quam dulcis, quam amara sapientibus. Qui, inquit, te diligunt te non cognoscunt. Ipsi te contempnunt qui te intelligunt. Fuge ergo (dicit Augustinus), sapiens, et curre, sapiens, quia sic erit vita tua quasi aliena." FM 328

Is saibhre fós sampla Mhic Aingil, mar a aithnítear oidhreacht na Gréige is na Róimhe:

Ní ghairionn an sgrioptúir dod bheathuidh achd bláth beag sheargas a mbeagán do laethibh, nó imirce as an mbroinn ndorcha gusan ttumba, nó néul beag sgaoiltear leis an nga ngréine, nó nead áilleóigi bristear le cloigh mbig thilgeas gárrlach ris, nó deatach beag théid gan moille ar neimhní, nó sgáile ar siobhal. Adeir Euripedes nach fuil innti achd aonlá amháin; 7 Demetrius Phal. gurab lór di moimint do chur 'na comparáid. Adeir Pláto nach foil innti achd suan duine 'dhúsgadh, 7 Chrisostomus nach foil innti ach aisling. SSA 30 
Cuid suntais é nach neamhchosúil leis seo an liosta samplaí a fhaightear ó easpag Protastúnach an Droma Mhóir blianta beaga ina dhiaidh seo:

... the wise men of the world have contended who shall best fit man's condition with words signifying his vanity and short abode. Homer calls a man 'a leaf', the smallest, the weakest piece of a short-lived unsteady plant. Pindar calls him 'the dream of a shadow'; another 'the dream of the shadow of smoke'. But St. James spake by a more excellent Spirit, saying 'Our life is but a vapour' ...

\section{Trí Bior-Ghaoithe an Bháis}

Is as cúlra na seanmóireachta a thagann dán cáiliúil an Chéitinnigh 'A Bhean lán de stuaim', agus faightear a mhacalla in FM, faoin gcuid sin de theagasc na heaglaise faoin ndrúis: De Luxuria. Mar seo a osclaíonn an chéad chuid 'De occasionibus luxurie' (ar ócáidí na drúise):

6. Circa autem eius occasiones est sciendum quod secundum quosdam quinque sunt occasiones inducentes in hoc versu contente:

Visus et alloquium, contactus et oscula, factum. FM 648

Níl anseo ach cuid den fhoirmle a bhí in úsáid go coitianta sa mheánaois:

Compages flagrantis quinque feruntur amoris:

Visus et alloquium, contactus et oscula amantum:

Postremus coitus, luctati clausula belli:

His in honore suo poterit desistere spado,

Ni temptare suum mavult post cepta pudorem. ${ }^{6}$

Caibidil a seacht de Liber Ecclesiasticus atá mar phointe tosaigh de thráchtas an Chéitinnigh ar an mbás:

7. Más eadh is é ollmhughadh is fearr dhó do dhéanamh air féin, bheith ag sgrúdadh 7 ag sgáthántacht 7 ag bioth-chuimhniughadh an bháis 7 shomharbhtachta an duine do shíor, do réir Solaimh san seachtmhadh caibidil in Ecclesiastico, mar a n-abair, memorare novissima etca. TBGB 7

Tá an téacs céanna ón mBíobla go mór i gceist chomh maith san FM:

De Memoria Mortis

Secundo humilitatem inducit memoria mortis, de qua Ieronimus ait: 'Facile,

${ }^{5}$ Jeremy Taylor, Rules and Exercises of Holy Dying (London, 1651).

${ }^{6} \mathrm{E}$. R. Curtius, European Literature and the Latin Middle Ages (London, 1953) (leagan Béarla), 512. 
inquit, contempnit omnia qui se cogitat moriturum.' 'Memorare novissima tua," iuxta consilium Sapientis, 'et in eternum non peccabis.' FM 96

Is é an téacs céanna a bhí mar phointe tosaithe chomh maith ag filí cráifeacha an 13ú haois, mar is léir ón sampla cáiliúil i gcló ag Carleton Brown i Lyrics of the 13th Century (lch 19).

Ceann de na samplaí coitianta ó thraidisiún na mbestiaria is ea an ceann seo a leanas:

8. Do bhríogh iomorra gurab do dhearmad do thabhairt i gcuimhne an bháis tig an duine do dhéanamh peacaidh, is eadh dhligheas do dhéanamh amhail do-ní an phéacóg. Is eadh cheana do-ní sí, an tan at-chí a hearr iol-dathach i n-eagar ós a cionn, 7 mhothuigheas dá bhríogh sin a huaill ag ardughadh, do-bheir silleadh síos ar a cosaibh garbha gartacha, 7 tig don mhíghné at-chí ar a cosaibh a macnas 7 a mómhaireacht do mhúchadh. TBGB 75

Secundo, quia male sua recogitans est bonorum morum occultatrix, sicut pavo aspiciens pedes turpissimos caudam suam dimittit lucentem. FM 64

\section{Ó thraidisiún na n-exempla an ceann seo a leanas:}

9. Quod eciam iudicium verum viguit apud eos narrat Valerius libro 6, quod cum quidam iudex male iudicasset, rex Cambices pellem eius de corpore extractam, sedili suo iudiciario, apposuit et filium eius in eo post eum iudicaturum sedere iussit, ut ipse ad memoriam revocaret quod propter falsum iudicium sic punitus erat pater suus. Unde quidam metrista ait:

Sedens in cathedra iustus iudex stabilis sta.

Sint tibi lucerna, lex, ius pellisque paterna.

A minibus reseces munus, ab aure preces. FM 500

Is mór idir an dá leagan agus níl an véarsa filíochta sa Ghaeilge.

Léaghthar sompla cosmhail ris so ag Ammianus, san .23. leabhar, ar rígh do bhí ar an bPersia, darbh ainm Cambyses. Do bhí iomorra breitheamh breabóideach, darbh ainm Sisamnes, ag an rígh se, rug claoin-bhreath mar gheall ar chumaoin fuair ó neach don dá rann do bhí i n-imreasan 7 an tan fuair an rí deimhin air sin, rug breith bháis ar an mbreitheamh, 7 fós tug fá-deara a chroiceann do bhuain de, 7 a chur mar chumhdach ar an gcathaoir bhreitheamhnais 'na gcleachtadh an breitheamh bheith, mar shompla dona breatheamhaibh oile do thiocfadh 'na dhiaidh, ionnus nach déandaois claonadh an reachta. TBGB 213-14

Is muinteartha fós, ná sampla 8 , an sampla seo: 
10 ... est sciendum quod ita contingit in fine de avaro divite et voluntario paupere sicut de gallina et falcone. Falco autem in vita in pugno portatur, in pertica collocatur, de carne recenti reficitur. Set gallina in locis vilissimis sicut in fovea et sterquilinio et huiusmodi querit refectionem, in tugurrio locatur. Et tamem mortuo falcone in foveam proicitur, et gallina assata coram rege et magnatibus in aulam defertur. FM 392

... féadtar na daoine móra 7 na fo-dhaoine do choimmeas ris an seabhac 7 ris an gcirc ré linn a mbeatha 7 d'éis a mbáis; do bhríogh go mbí onóir ar an seabhac ré linn a bheatha 7 go dteilgthear san chamra i ndiaidh a bháis é. An chearc iomorra, léigthear san laithigh dhi ré linn a beatha, ag buain oileamhna as an aoileach. Gidh eadh, an tan marbhthar í, do-níthear a hollmhughadh go honórach, go gcuirthear ar bhord na n-uasal í. TBGB 32

Ó thraidisiún na dtráchtas leighis a eascraíonn móitíf na 'Proprietates Mortis', Comharthaí an Bháis, catalóg de chomharthaí an bháis, a úsáidtear sna téacsanna cráifeacha d'fhonn daoine a bhrostú chun aithrí:

11. Et nota quod signa mortis secundum beatum Ieronimum sunt hec: Quando nasus frigescit, facies pallescit, oculi tenbrescunt, aures surdescunt, nervi et vene rumpuntur, cor in duas partes dividitur. Nichil vilius nec abhominabilius cadavere mortuo. In domo non permittitur ne eius fetore familia moriatur. In aere non suspenditur ne aer inficiatur. In aqua non proicitur ne illa corrumpatur. Set quid? Revera terra foditur et in terram proicitur et tamquam venenum mortiferum, ne amplius compareat, a terra cooperitur. Unde quidam Anglice sic dicit ... FM 718

Ina léirmheas cuimsitheach ar The World of Geoffrey Keating le Bernadette Cunningham, Éigse 25 (2005), 136-49, deir Cathal Ó hÁinle [140], 'In addition to providing a synopsis of the content of TBB (48-9), the author also discusses the biblical, medieval and contemporary sources of the work (50-57). However, she does not advert to Keating's citation of some thirty-four stanzas from Irish syllabic poems in support of various points of teaching in his text. By adding this further layer to the typical panoply of biblical, patristic and medieval reference of European catechetical literature, Keating heightened the Irish flavour of his text and, furthermore, demonstrated his respect for the authority of the native literary tradition and provided evidence of his having received some significant education in a bardic school'. Ach faoi mar a thuigtear ón sampla thuas, agus tríd síos san FM, ba nós coitianta é sna tráchtais chráifeacha é pointí teagaisc a léiriú le véarsaí filíochta.

... do-chífe an corp ar gcruadhughadh 7 ar gcomhfhuaradh, na baill ar siabhradh

7 ar searghadh .i. na lámha ar gcrapadh 7 ar gcaolughadh, na cosa 'na spairtibh 
splíonuighthe amhail easglanna iomthroma, an béal arna bhán-ghormadh 7 an déad arna dubhadh, na gruaidhe arna nglas-bhánadh 7 na súile arna slogadh, an t-éadan 7 an aghaidh ar n-odhradh, an tsrón arna caolughadh 7 arna cuasdúnadh, na clausa ar dtanughadh 7 ar dteimhliughadh, an folt ar dtothlughadh 7 ar dtuitim ... TBGB 37

\section{Agus Leopold Bloom Joyce ag machnamh ar an mbás, deir sé:}

The death struggle. His sleep is not natural. Press his lower eyelid. Watching is his nose pointed is his jaw sinking are the soles of his feet yellow. (Ulysses, 149)

Cuid de mhóitíf uilechoitianta an ubi sunt is ea an sampla seo a leanas:

12. Unde Ieronimus: 'Non es, inquit, David sanctior, non es Salomone sapiencior, non Absolone pulcrior, non Sampsone forcior.' Et tamen omnes isti a mulieribus fuerunt decepti. FM 706

Óir níor chaomhain a cheannas feadhna Séasair, ná a neart Samson, ná a eagna Solamh, ná a áitheas Alexander, ná a áille Absolón, ná a intleacht Aristotail, náid a bhriathra bláithe briocht-shnoighthe Cicero, ná aon oile aon-acfuinn dá mbí ann, ar ghuaisibh an bháis. TBGB 22

Is i PnamB, áfach, is túisce a fhaighimid leagan fileata den ubi sunt áirithe seo, agus is díol suntais gur iontuigthe as gur sagart a thiontaigh an bhunfhoinse Laidine go Gaeilge, rud a b'fhéidir a thaispeáint, creidim, faoi chuid mhaith de litríocht na gaoise san 17 ú haois:

... 7 atá a fhios aguibh gur lé mnáibh do mealladh 7 do milleadh Sampson, Dáth Rígh, Salamon, 7 Peadair Easbol, 7 morán do dhaoinibh eile cumasacha do réir mar adeir an file:

Adam, Sampsonem, Petrum, Davidem, Salomonem, Decepit mulier; quis modo tutus erit.

Ag so mar do cuireadh an ní céanna a nGaoidheilg lé sagart suairc, sáimheólach, i. Donnchadh Mhac Cárrtha, atá ainnis a ccúram poróiste Theampuill Muire a cCorca:

Ó mealladh lé mnaoi Dáth Rígh 7 Solamon glic,

Sampson is na mílte saoi budh calma a ngoil, is ár $\mathrm{n}$-athair do suígheadh go haoibhinn a bParrathas tsoir, is Peadair; a Chríost, cá díon dá bhfearaibh-ne dul? TBGB $53^{7}$

${ }^{7}$ Faightear an véarsa seo ina dhiaidh sin sna Búrdúin Bheaga (eag.) T. F. O'Rahilly (Baile Átha Cliath, 1925), i dteannta mórán de véarsaí eile PnamB. Deir an fear eagair faoi na búrdúin seo 'gur sgáthán iad ina bhficimíd croí agus smaointe na 


\section{Párliament na mBan}

Tráchtas ar na suáilcí agus ar na duáilcí, seánra a bhfuil sinsearacht fhada laistiar de i dtraidisiún na caiticéise is ea PnamB agus is as na foinsí comónta seanmóireachta, fáiscthe as taithí laethúil an duine, don chuid is mó, atá a húdar agus údar an FM ag tarrac. Ó chúrsaí ceardaíochta an léiriú acu beirt ar pheaca na drúise:

'Is mór a' peaca an drúis', ar sí, '7 ní bhfuil ann acht teine bhíos ar dearglasa, 7 ní hé Dia aduighios an teine sin acht an diabhal. 7 fé mar do-ní an gobha an uair nách fiadann sé an t-iarann do shásúghadh do réir a thoile, cathann sé annsa teine é 7 bíonn sé a' séide na mbolg nó go mbíonn an t-iarann teith 7 ann sin ceartaighionn sé é mar is toil leis féin, sin mar bhíos an diabhal. PnamB 39-40

Nota eciam quod diabolus ut hominem alliciat ad hoc peccatum, facit ad modum fabri. Qui cum non potest ducere ferrum ad libitum, ad ignem ponit et fortiter sufflat. Sic diabolus quando non potest hominem ducere ad libitum suum, primo accendit circa illum ignem voluptatis. FM 697

\section{Tagann sampla eile fós sna sála ar sin ó chúrsaí cócaireachta:}

14. Samhaltar é ré crocán ar an adhbhar, dá ghile bhíos an crocán, má curthar ar an ttine é bia sé dubh ... Farrais sin, an crocán bhíos ag fiucha, muna mbiaidh ann acht aonghráinne amháin póinire, tógbhann an fiucha a n-uachtar é 7 taisbéann é. Mar sin bhíos na daoine drúisiúla ... PnamB 40

Verbi gracia: si enim olla iuxta ignem ebulliat, ne effundatur quod in ea continetur, tunc enim unum istorum trium fieri consuevit, quia vel aqua frigida infunditur, vel de lignis subtrahitur, vel ipsa olla ab igne elongatur. FM 656

Ó chúrsaí iascaireachta na samplaí léirithe i gcás na leisce ag an bheirt seanmórithe:

15. Do-címaoid an t-uisce ná corruíghionn 7 nách bíonn rith aige go loghann sé. PnamB 43

Nam sicut aqua non mota ex se putrescit... FM 661

7 as amhlaidh do-ní an diabhal faoi mar do-ní an t-iasgaire do shailíghios an t-uisge chum nách faicfeadh an t-iasg an líon 7 go rachadh sé ann asteach. PnamB 44

In aqua autem turbida melius piscatur quam in clara. FM 459

ngnáthdhaoine ón seachtú aois déag anuas'. Is dóichí, dar liom, gur iasachtaí ón Laidin a bhformhór, a chuir sagairt iarThriontacha a oileadh ar an mór-roinn in oiriúint dá bpobal ar fhilleadh abhaile dóibh. 
Ó thaithí na feirmeoireachta sampla an chathaithe a leanas:

16. As iad na mná bhíos mímhacánta mar seo chruinnigheas armáil an diabhuil, faoi mar do-ní an muicidhe an uair bhíos a mhuca fánach diombaileach ar fud na ccnoc, beirionn sé ar mhuic dhíobh 7 bainionn sgreadach aisde, 7 leis sin cruinníghid an chuid eile. Sin mar do-ní an diabhal. PnamB 54

... non debebus vestibus placere set moribus. Set hoc non intendunt iste fatue mulieres que in solempnitatibus vestibus luxuriosis tam in capite quam in corpore ad peccatum excitandum se ornant. Et ideo sicud custos porcorum qui illos disperses vult congregare unum facit clamare et statim alii conveniunt, sic revera dyabolus quando suos intendit congregare unam de filiabus suis facit clamare ... FM 50

Baintear casadh eile fós as an dtaithí chéanna i ndearadh fhear na cúlchainte, á chomhshamhlú le priompallán an Chéitinnigh:

17. Deallraighthear fear na cúlchainnte ré muic ar an ábhar an uair théid an mhuc asteach a ngáirdín 7 do-chí sí luibheanna breaghtha ar thaobh di 7 salachar an an ttaobh eile, ritheann sí chum an tsalachair 7 ní chuirionn beann ar na luibheannaibh ná ar na bláthaibh suairce bhíos ann. PnamB 56

\section{Samhlaítear an bladaire le camelion agus leis na sirenes:}

18. Samhlaighthior an bladaire lé hainmhídhe darab ainm camaelion noch ghlacus air féin gach aontsórd datha bíos ar an áit iona mbíonn sé. Mar sin do-ní an bladuire léna chómhráidhtibh fallsa meallta ganguideach' líon do leatha fé chosaibh a chómharsan. PnamB 48

... adulator bene potest comparari cuidam animali dicto Gamaleon, de quo Ysidorus Ethimologiarum libro 12 dicit quod est quoddam animal vivens in aere, cuius color variatur ad variacionem obiectorum quibus applicatur. FM 173

19. An uair bhíos deithneas ar dhuine ag dul dá dhúithe is cóir do cluas bhoghar do bheith aige ag imtheacht tar cheól marbhthach na murrúghach, i. sirens nó mermaids. PnamB 49

Syrene autem sunt monstra marina, que ut dicitur dulcedine sui cantus nautas ad se attrahunt et submergunt...Secundum tamen veritatem fuerunt meretrices que transeuntes ducebant ad egestatem, ut dicit Ysidorus. FM 573

Téama tábhachtach de chuid na meánaoise ab ea an eicsigéis ar an dá shliocht seo a leanas sa Bhíobla: 'Intravit Jesus in castellum' ( $L u ́ c, \mathbf{x}$, 38) agus 'Ite in castellum quod contra vos est' (Matha, xxi, 2). Tá dílsiú foirfe déanta ag an gCéitinneach agus ag an gColmánach ar an nós seo sa léamh fáithchiallach acu beirt sna samplaí cumasacha seo a leanas, atá chomh léannta cruthaitheach leis an dá shampla dheireanacha ón FM: 
20. Meas féin, a dhuine thruaigh, créad an t-uathbhás 'na mbiadh caiptín nó ceann feadhna mar aon ré beagán buidhne i gcaisléan éadaingean éislinneach, 7 tromshluagh tairptheach tréan-aidhbhseach i bhfoslongphort réna ucht, 7 a mhuinntear arna marbhadh, 7 urmhór an tsluaigh ar dteacht ar aon-láthair ris, 7 a n-airm nocht aca; 7 baránta borb baoith-chéillidhe rompa lán do chonfadh catha, 7 caiptín an chaisleáin gan anacal air, ná dáil chabhra 'na chionn. Mar an gcéadna don anam bhíos ag bárdacht chaisléin chriadhamhail an chuirp, taobh ré beagán buidhne i. ris na céadfadhaibh, an tan tig trom-shluagh na dtaom 7 na tinneas, na ngalar agus na n-easláinteadh, mar aon ris an gcaiptín mbríoghmhar mboirb-neimhneach .i. ris an mbás, 7 shuidhid i bhfoslongphort réna ucht, 7 lingid tar múraibh na mball 7 tar comhladhaibh na gcéadfadh, go soichid compar an chroidhe, mar a mbí an t-anam. Feannaid 7 foghaid, díbrid 7 míochóirighid a mhuinntear 7 a bhean phósta ar a chomhair, i. a chorp 7 a chéadfadha. PnamB 36-7

Is neimhiongnadh fós an cholann do bheith neimhbríoghmhar do bhríogh nach fuil acht long ladrann 7 caisléan coinbhliochta innte. Óir ní bhí réidheachadh ná ró-bháidh ar dtús idir sí féin 7 an t-anam, do réir Phóil, san chúigeadh caibidil go Galatas, mar a n-abair, caro concupiscit adversus spiritum 'mianuighidh an cholann i n-aghaidh na hanma.' PnamB 93

An uair is maith lé duine caisleán do ghabháil brosdaighionn sé chum doruis an chaisleáin dá shealbhúghadh. As í an sgórnach an dorus 'na ttéid an craos 7 armáil an diabhuil asteach, 7 an té agá mbíonn dorus an chaisleáin is urusa do an caisleán do bheith aige. Dá bhrígh sin is ar gheata an bhéil 7 na sgórnuíghe is cóir an ghárda is mó do chur, ar an adhbhar, muna ccurthar srian ris an gcraos a' chéad uair, ní bhfuil acht díomhaoineas cur a n-aghaidh na ndubháilcídhe eile. (PnamB 37)

Samhaltar an duine leisgeamhuil nó lorgánta le caisleán ar a mbí mórán tiaighearnaoi air noch aigeá ccaillionn an té bhíonn leasg faillítheach a chuid don chaisleán go hiomlán ag duine eile dá sgiuba uaidh. PnamB 42

Est autem sciendum quod ad veram pacem servandam necesse est facere sicut quando aliquod castrum inpugnatur ab aliquo magno, cui dominus castri non poterit resistere nisi securius ordinaverit de castro et maxime ab intra: necessarium est primo ut consideret utrum in castro sint aliqui pacem illius perturbantes; secundo si quos tales invenerit, debet eos extra castum suum eicere et de illo iusticiam facere; tercio debet castrum suum fortiter munire, ita scilicet quod non habent necesse extra illud ire; quarto debet ipsum firmum et clausum tenere. Revera sic ex parte ista, quia enim vulgariter dicitur: 'Bonum castrum bene custodit qui cor suum bene custodit.' FM 142-4

Exemplum: Sicut autem castrum faciliter capitur mortuo ianitore et hostibus introductis, sic devicta carne, que ianitor est luxurie, per quam ceteri hostes, hoc est omnes carnis motus illiciti, introducuntur, castrum anime, idest mundicia vite per quam celum rapitur, facillime capietur. Et sicut castrum bene muratum et nulla parte confractum securitatem dat inhabitantibus, sic corpus humanum continencia bene munitum omnes voluptates expellit et anime securitatem prestat contra omnes hostes. Unde pro facili devincit expugnacionem istorum hostium, scilicet carnis, mundi, et demonis. FM 720 
Tá sé le tuiscint ón gcomparáid seo idir samplaí ó mhórshaothair chráifeacha an 17ú haois sa Ghaeilge agus an FM go rabhthas ag leanúint de thraidisiún sheanmóireacht na meánaoise agus é sin á chur in oiriúint do riachtanais chaiticéise na linne. Ar a shon gur féidir cuid mhaith de chorpus na Gaeilge a shíneadh lena macasamhail ag recusants an Bhéarla, is cuid suntais é a bheith chomh mór sin i dtuilleamaí lámhleabhair seanmóireachta na meánaoise agus traidisiún na scéalaíochta eiseamláirí. Míniú amháin air seo is ea go raibh reitric seo na seanmóireachta ag teacht le dúchas na Gaeilge agus ag freagairt do thoscaí stairiúla agus do riachtanais chaiticiostacha na linne ag an am gcéanna.

\footnotetext{
Noda

ESA O'Brien, Patrick, Eochair-Sgiath an Aifrinn (Baile Átha Cliath, 1898).

FF Comyn, David agus Patrick Dinneen (eag.), Forus Feasa ar Éirinn (4 iml., Baile Átha Cliath, 1902-14).

FM Wenzel, Siegfried (ed.), Fasciculus Morum: A Fourteenth-Century Preacher's Handbook. 1989.
}

SSA Aodh Mac Aingil, Scáthán Shacramuinte na hAithridhe (Lobháin, 1618), Cainneach Ó Maonaigh (eag.) (Baile Átha Cliath, 1952).

PnamB · Dónall Ó Colmáin, Párliament na mBan, 1670. Brian Ó Cuív (eag.) (Baile Átha Cliath, 1977).

TBGB Séathrún Céitinn, Trí Bior-Ghaoithe an Bháis, $c .1631$. Osborn Bergin (eag.) (Baile Átha Cliath, 1931). 